\title{
Robust UAV Aided Data Collection for Energy-Efficient Wireless Sensor Network with Imperfect CSI
}

\author{
Xiaoying Fu, Yi Cen*, Huilan Zou* \\ School of Information Engineering, Minzu University of China, Beijing, China

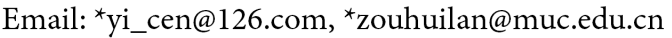

How to cite this paper: Fu, X.Y., Cen, Y. and Zou, H.L. (2021) Robust UAV Aided Data Collection for Energy-Efficient Wireless Sensor Network with Imperfect CSI. Journal of Computer and Communications, 9, 109-119.

https://doi.org/10.4236/jcc.2021.95008

Received: April 21, 2021

Accepted: May 28, 2021

Published: May 31, 2021

\begin{abstract}
Due to its air superiority and high mobility, unmanned aerial vehicle (UAV) can obtain better line-of-sight (LoS) link transmission channel. Therefore, UAV assisted data collection for wireless sensor networks (WSNs) has become an important research direction. This paper intends to minimize the loss of WSNs for the robust data acquisition and communication assisted by UAV under the imperfect channel state information (CSI). On the premise of ensuring the completion of the communication task, we jointly optimize the wake-up schedule of SNs and the flight trajectory of the UAV, by considering the flight speed of the UAV and the sparse access of all sensor nodes (SNs) in WSN. Because the formulated optimization problem is a mixed integer nonconvex problem, we decompose the original problem into the efficient suboptimal solutions to overcome the difficulty of the optimization. Finally, the number of access node corresponding to the optimized operation time and access efficiency is induced for the entire WSN system efficiency improving. The simulation shows the performance gains of our proposed scheme and the influences of the system parameters are analyzed.
\end{abstract}

\section{Keywords}

UAV, Data Collection, Wireless Sensor Network, Trajectory Optimization, Sparse Access

\section{Introduction}

Wireless sensor networks (WSNs) are usually composed of a large number of low-cost sensor nodes (SNs). Since the extensively deployed SNs are usually po-

${ }^{*}$ Corresponding author. 
wered by limited energy sources, it is difficult to recharge them when exhausted. Noticed its high mobility and flexibility, Unmanned Aerial Vehicle (UAV) is widely used in military and civil fields, such as search and rescue, air surveillance, data packet transmission [1] [2]. It is achievable to improve WSNs' energy saving ability by UAV aided. Generally, there are three applications of UAV in wireless communication: the first is as an air base station to supplement the service area that the ground base station cannot cover. Some papers studied The best location of the UAV [3] and the corresponding path loss model that simultaneously applies line-of-sight (LoS) and non line-of-sight (NLoS) conditions are studied [4]. In [5], the influence of the altitude and trajectory of the UAV on the coverage rate of the system in two scenarios in the D2D communication network is considered. The second one is to provide connection for remote users without direct connection between users and base stations, as an air relay. In order to maximize the end-toend throughput between the source node and the destination node, the multi-hop and the single-hop UAV relay systems are mainly considered [2] [6]. Besides, applying UAV as a flight access point for data collection and information dissemination has become an attractive field of research [2] [7]-[12].

Because WSN has the characteristics of a large number of node deployments and large-scale data sampling, the data collection strategy based on UAV access is more suitable for large-scale WSN scenarios. In [7], the main consideration is to minimize the transmission energy consumption by optimizing the ground node transmission strategy and the trajectory of the UAV. When the UAV collects data, in addition to the transmission loss, there is also the power consumption of the UAV flying. [8] presents optimization problems that minimize the weighted sum of two consumption. In [9], the trade-off between the ground node transmission loss and the UAV flight loss for two practical UAV flight trajectories (circular flight and straight flight) is discussed. While [10] is for the flight trajectory planning, the scheduling and power allocation strategy of the ground nodes and the UAV are jointly optimized. In addition to the above-mentioned case of data collection by a single drone, there is also a multiple drones case for data collection on a group of nodes. [11] discusses the relationship between multiple drones and one set of nodes, so as to the problem of the trade-off between the aviation cost and the ground cost between the group of sensor nodes. In the entire data collection process, the time of data collection is also very important. Therefore, in [12], the trajectory and the height of the UAV are directly considered to minimize the total task time.

In view of the problem of data collection between multiple nodes and drones, there still exist several short comings at this stage:

1) Often in real life, the distribution of ground nodes is relatively concentrated and random. But the current research is mainly for a small number of scattered SNs. Due to the centrality of SNs distribution, it should be multi-access, however it only considers the single node access scenario.

2) In the current papers, the optimization time problem mostly only considers the time for UAV to collect data, but not consider the entire time required for 
the target problem planning, including computing time etc.

In this paper, we mainly study the data collection between multiple randomly distributed ground nodes and UAV. On the premise of ensuring the completion of the communication task under the imperfect channel state information (CSI), UAV trajectory and ground node wake-up scheduling plan are jointly designed and deployed to reduce the loss of ground node. Especially, we focus on the multiple nodes deployed densely enough on the ground are sparsely connected to the UAV for data transmission. In addition, the optimization time and efficiency performances are also studied via varying the numbers of the connecting ground sensor nodes at the same time. The best overall efficiency is given through simulation.

\section{System Model}

We consider the UAV assisted wireless sensor network shown in Figure 1, where the UAV is responsible for collecting data from SNs. Suppose there are KSNs on the ground, denoted as $\left\{s_{k}, 1 \leq k \leq K\right\}$, and the horizontal position are randomly distributed as $x_{k} \in R^{2 \times 1}$. Denote the position of the $k$-th $\mathrm{SN}$ as $\left[x_{k}{ }^{T}, 0\right]^{T}$. The UAV flies at a fixed height of $H \mathrm{~m}$, and the maximum flying speed is $V_{\max } \mathrm{m} / \mathrm{s}$. The trajectory projected by the UAV on the ground is assumed to be $q(t), q(t) \in R^{2 \times 1}$, where the position of the UAV can be expressed as $\left[q(t)^{T}, H\right]^{T}$. Let, $\|\vec{h}\|=h,\left\|\vec{y}_{k}(t)\right\|=q(t)-x_{k}$, assume that there is a slight disturbance to the UAV's altitude and trajectory during flight, denoted as, $\Delta \vec{h}(t)$, $\Delta \vec{q}(t)$, where, $\|\Delta \vec{h}(t)\| /\|\vec{h}\|<\varepsilon_{1},\|\Delta \vec{q}(t)\| /\left\|\vec{y}_{k}(t)\right\|<\varepsilon_{2}, \quad \varepsilon_{1}, \varepsilon_{2}<<1$.

During the flight, it is assumed that its initial point and end point are fixed, expressed as $q_{0}, q_{F}$, and satisfied $q_{0}, q_{F} \in R^{2 \times 1}$. The time of the UAV in the entire data collection process is $T$, and each ground node generates $S_{k}$ bits of data within this time. It can be seen that the UAV should satisfy at least the following formula during the flight: $\left\|q_{F}-q_{0}\right\| \leq V_{\max } T$, so that there is at least one path that satisfies the full flight from the initial point to the end point on the basis of the maximum speed and mission time. In order to facilitate the overall optimization, the entire time $T$ is divided into $M$ time slots, and the length of each time

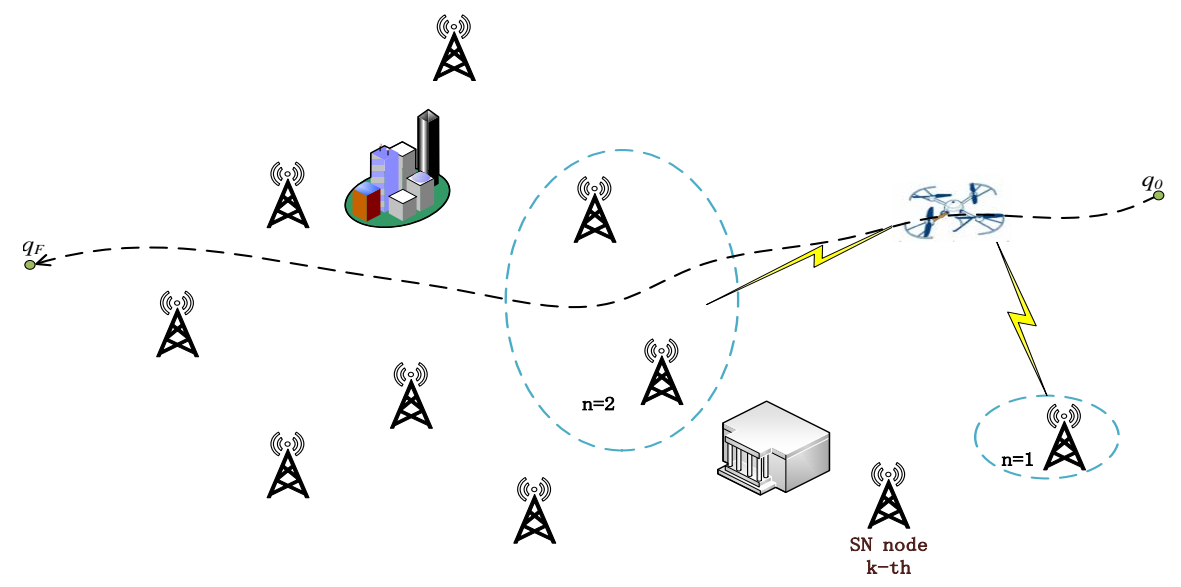

Figure 1. System model diagram. 
slot is $\Delta t$, so there is $T=M \times \Delta t$. As far as possible, the $\Delta t$ can be made infinitely small. Then the position of the UAV in each flight time slot can be regarded as unchanged. Therefore, the trajectory of the UAV can be regarded as a sequence that is $\{q[m], 1 \leq m \leq M\}$. Assuming that the maximum distance traveled by the drone in the time interval is $D_{\max }$, where $D_{\max }=V_{\max } \times \Delta t$.

During the duration $T$, in order to avoid the waste of energy due to idle monitoring of SNs, each SN is awakened by the UAV. Define a variable $a_{k}(t)$ to represent the wake-up and sleep relationship of the $k$-th SN at time $t$, which satisfies $a_{k}(t) \in\{0,1\}$. When $a_{k}(t)=1$, it means that the $k$-th $\mathrm{SN}$ is in the awakened state at this time, and transmits data to the UAV. In order to avoid mutual interference when multiple SNs transmit data to the UAV at the same time, we assume that the communication system uses orthogonal frequency division multiplexing (OFDM) technology, and the SNs occupy different frequency bands to upload data to the UAV separately. According to the unmanned track coordinates and the horizontal position of the SNs, the real-time distance between them can be expressed as:

$$
d_{k}[m]=\sqrt{\left\|\vec{y}_{k}[m]+\Delta \vec{q}[m]\right\|^{2}+\|\vec{h}+\Delta \vec{h}[m]\|^{2}}, \quad 0 \leq m \leq M .
$$

It is assumed that the link from the SNs to the UAV to upload data is a quasi-static block fading channel, where the channel remains unchanged in each fading block and may change between fading blocks. The duration of each fading block is usually much smaller than $\Delta t$. Therefore, we denote the number of fading blocks in each time slot as $S$. In fact, $S$ is much larger than 1. Under the general fading channel model, the channel coefficient of the $s$-th fading block in time slot $\mathrm{m}$ between the UAV and the $k$-th SN can be modeled as $h_{k}[m, s]=\sqrt{\beta_{k}[m]} \tilde{h}_{k}[m, s]$, where $\beta_{k}[m]$ represents the large-scale channel attenuation, and this value only depends on the distance between the UAV and the $\mathrm{SN}, \quad \tilde{h}_{k}[m, s]$ represents the small-scale channel attenuation. The large-scale channel attenuation can express in time slot $\mathrm{m}: \beta_{k}[\mathrm{~m}]=\beta_{0} d_{k}^{-\alpha}[\mathrm{m}]$.

Among them, $\alpha$ is the path loss index. In general, its value is $\alpha \geq 2$, and $\beta_{0}$ represents the power gain of the reference channel when the distance is 1 meter. Small-scale channel attenuation is considered to be an independent and uniformly distributed random variable, so it satisfies $E\left\{\left|\tilde{h}_{k}[m, s]\right|^{2}\right\}=1$. Because the distance between the UAV and the SN in each small time slot is regarded as constant. Therefore, the channel coefficient is the same in the same time slot, but are changed in different time slots. By confirming the UAV's trajectory, wake-up scheme, and transmission rate, the UAV notifies the optimized transmission rate on the time slot through the downlink control link. For the $k$-th sensor, the achievable rate on the $s$-th fading block of time slot $\mathrm{m}$ can be expressed as:

$$
C_{k}[m, s]=\log _{2}\left(1+\frac{\left|h_{k}[m, s]\right|^{2} P_{k}}{\sigma^{2} \Lambda}\right) .
$$

where, $\sigma^{2}$ represents the noise power, and $\mathrm{P}_{\mathrm{k}}$ represents the transmission power of the $k$-th SN to send data to the UAV. $\Lambda$ is the signal-to-noise ratio differ- 
ence between the actual modulation scheme and the theoretical Gaussian signal. Considering the inter-ruption probability between the UAV and the $\mathrm{SN}$, assuming that $R_{k}[\mathrm{~m}]$ represents the transmission power, and the probability of different fading blocks in each time slot is the same, the $k$-th SN can be interrupted at time $\mathrm{m}$ and the probability is expressed as follows:

$$
p_{k}[m]=P\left(C_{k}[m, s]<R_{k}[m]\right)=P\left(\left|\tilde{h}_{k}[m, s]\right|^{2}<\sigma^{2}\left(2^{R_{k}[m]}-1\right) \Lambda / \beta_{k}[m] P_{k}\right)
$$

According to the definition of the cumulative distribution function, the above formula can be transformed into the following formula:

$$
P_{k}[m] \cong \operatorname{Pr}\left(\frac{\sigma^{2}\left(2^{R_{k}[m]}-1\right) \Lambda}{\beta_{k}[m] P_{k}}\right) .
$$

The $P r$ function represents the cumulative distribution function related to $\left|\tilde{h}_{k}[m, s]\right|^{2}$, and the probability function is a non-decreasing function related to the transmission power. In order to complete the transmission task of each SN, the transmission power under the maximum tolerable probability should be able to finally meet the task requirements. $P_{k}[m]=\kappa$, it can be expressed as follows:

$$
R_{k}[m]=\log _{2}\left(1+\frac{\operatorname{Pr}^{-1}(\kappa) P_{k} \beta_{0}}{\sigma^{2}\left(\left\|\vec{y}_{k}[m]+\Delta \bar{q}[m]\right\|^{2}+\|\vec{h}+\Delta \vec{h}[m]\|^{2}\right)^{\alpha / 2} \Lambda}\right) .
$$

where $\kappa$ is the maximum tolerable interruption probability, and the $P r^{-1}$ function is the inverse function of the $\operatorname{Pr}$ function.

\section{Problem Formulation}

In order to ensure the fairness of SNs in terms of energy consumption, minimizing the maximum energy consumption of SNs is chosen. Let $A=\left\{a_{k}[m], \forall k, m\right\}$, $Q=\{q[m], \forall m\}$. Our goal is to jointly optimize the wake-up plan $A$ and the UAV's trajectory $Q$ to minimize the maximum energy consumption of all $\mathrm{SNs}$, while ensuring that sufficient information can be obtained from the SNs. $B$ is the bandwidth of the channel, the power in the time interval can be expressed as: $E_{k}=\Delta t P_{k}$. The entire optimization problem can be written as follows:

$$
(P 1): \quad \min _{X, Q, \theta} \theta
$$

$$
\begin{aligned}
& \text { s.t. } \sum_{m=1}^{M} a_{k}[m] E_{k} \leq \theta, \quad \forall k \text {, } \\
& \sum_{m=1}^{M} a_{k}[m] R_{k}[m] \geq r_{k}, \quad \text { where } \sum_{m=1}^{M} a_{k}[m] \neq 0 \quad \forall k, \\
& \sum_{k=1}^{K} a_{k}[m] R_{k}[m] \geq r_{m} \quad \forall m, \\
& \sum_{k=1}^{K} a_{k}[m] \leq n \quad \forall m, \\
& a_{k}[m] \in\{0,1\}, \\
& \forall k, m, \\
& q[1]=q_{0}, q[M]=q_{F}, \\
& \|q[m]-q[m-1]\| \leq D_{\max }, \quad \forall m \geq 2 .
\end{aligned}
$$

Here, the constraint variable $\theta$ is used to represent the minimized maximum energy consumption, where the constraint (6) ensures that the energy consump- 
tion of each sensor node does not exceed $\theta$. Constraint ( 7 ) is to ensure that the data volume goal of each ground node can be achieved. Constraint (8) is to make all the data collected by the UAV reach a certain value at the same time. Constraints (9) and (10) indicate the SNs scheduling scheme. At the same time, the number of ground nodes connected to the drone to upload data is not More than $n$. Constraints (11) and (12) constrain the UAV's speed, initial and final position. Since $P 1$ is a mixed integer non-convex problem, it is usually difficult to get the optimal solution. Therefore, in this paper, the main goal is to obtain an effective suboptimal solution of $(P 1)$.

\section{1) Problem reconstruction:}

In order to reconstruct the binary wake-up scheduling variable, it should be noted that there are a total of $S^{\star} M$ fading blocks in the time range $T$. If the solution $A$ of the relaxation problem is not binary, We can assign $N_{k}[m]$ fading blocks to any time slot $m$ of the ground node. Where,

$N_{k}[m]=\left(\tilde{N}\left|\min _{\tilde{N}}\right| \tilde{N}-S a_{k}[m] \mid, \tilde{N} \in Z\right)$ The value represented by $\tilde{N}$ is the nearest integer to $S a_{k}[m]$. When $S$ is large enough, the gap between $N_{k}[m]$ and $S a_{k}[m]$ is practically negligible. After relaxing the binary constraint in (10) to $0 \leq a_{k}[m] \leq 1$, the optimization problem can be described as follows:

$$
\begin{array}{rrr} 
& (P 1): \quad \min _{X, Q, \theta} \theta & \\
\text { s.t. } 0 \leq a_{k}[m] \leq 1, & & \forall k, m,
\end{array}
$$

$$
\text { (6), (7), (8), (9), (10), (11), (12) }
$$

\section{2) Decompose $P 1$ into two sub-problems:}

a) For any given trajectory $Q$, the optimal relaxation wake-up scheduling problem can be obtained by solving the following standard linear programming:

$$
(P 2): \min _{X, Q, \theta} \theta \quad \text { s.t. (6), (7), (8), (9),(13). }
$$

Suppose that in the 1-th iteration, the generated ground node scheduling scheme and the flight trajectory of the UAV are expressed as: $A^{l}$ and $Q^{l}$ respectively. During the 1 -th iteration operation, the transmission power can be expressed as:

$$
R_{k}[m]=\log _{2}\left(1+\frac{\operatorname{Pr}^{-1}(\kappa) P_{k} \beta_{0}}{\sigma^{2} \Lambda\left(\left\|\vec{y}_{k}^{(l-1)}[m]+\Delta \vec{q}^{(l-1)}[m]\right\|^{2}+\|\vec{h}+\Delta \vec{h}[m]\|^{2}\right)^{\alpha / 2}}\right)
$$

b) For any given wake-up plan $A$, optimize the UAV's trajectory to maximize the weighted minimum of the communication throughput of all ground nodes, where the weight is inversely proportional to $r_{k}$. Due to the existence of nonconvex constraints, an effective approximate solution can be obtained through continuous convex optimization technology [13], and it is guaranteed to converge to at least one local optimal solution. The main idea of this method is to maximize the lower bound of the transmission power in each iteration. It ensures the robustness of the communication system by limiting the lower bound of the transmission rate. The problem can be described as: 


$$
\begin{gathered}
(P 3): \quad \max _{Q, \varsigma} \min _{\Delta \bar{h}, \Delta \bar{q}} \varsigma \\
\text { s.t. } \quad \frac{1}{r_{k}} \sum_{m=1}^{M} a_{k}[m] R_{k}[m] \geq \varsigma, \quad \forall k,
\end{gathered}
$$

To decompose the $P 3$, the lower limit of the transmission rate needs to be changed first. Transform the formula (5):

$R_{k}[m] \approx \log _{2}\left(1+\frac{\operatorname{Pr}^{-1}(\kappa) P_{k} \beta_{0}}{\sigma^{2} \Lambda\left(\left\|\vec{y}_{k}[m]\right\|^{2}+2\left\langle\Delta \vec{q}[m] \cdot \vec{y}_{k}[m]\right\rangle+\|\vec{h}\|^{2}+2\langle\vec{h} \cdot \Delta \vec{h}[m]\rangle\right)^{\alpha / 2}}\right)$

Among them, because $\|\Delta \vec{h}(t)\|^{2},\|\Delta \vec{q}(t)\|^{2}$ are very small, they can be ignored. It can be seen that the transmission rate is a decreasing function about $\Delta \vec{h}(t), \Delta \vec{q}(t)$. When $\Delta \vec{h}(t)=\varepsilon_{1}\|\vec{h}\|, \Delta \vec{q}(t)=\varepsilon_{2}\left\|\vec{y}_{k}(t)\right\|$, it can obtain the lower limit of the transmission rate. Let $\delta_{k}(q)=\left\|q[m]-x_{k}\right\|^{2}, \quad \delta_{k}\left(q^{l}\right)=\left\|\vec{y}_{k}[m]\right\|^{2}$, $\gamma=\frac{1+2 \varepsilon_{1}}{1+2 \varepsilon_{2}}, \quad \Gamma=\sigma^{2} \Lambda\left(1+2 \varepsilon_{2}\right)^{\alpha / 2}$. Then:

$$
R_{k}[m]=\log _{2}\left(1+\frac{\operatorname{Pr}^{-1}(\kappa) P_{k} \beta_{0}}{\sigma^{2} \Lambda\left(1+2 \varepsilon_{2}\right)^{\alpha / 2}\left(\gamma H^{2}+\left\|\vec{y}_{k}[m]\right\|^{2}\right)^{\alpha / 2}}\right) . . \text { Through the first-order }
$$

Taylor expansion, the lower bound of $R_{k}[\mathrm{~m}]$ can be expressed as:

$$
\begin{aligned}
& R_{\mathrm{kl}}^{\mathrm{inf}}[m]=\log _{2}\left(1+\frac{\operatorname{Pr}^{-1}(\kappa) P_{k} \beta_{0}}{\left(\gamma H^{2}+\delta_{k}\left(q^{l}\right)\right)^{\alpha / 2} \Gamma}\right) \\
& -\frac{\operatorname{Pr}^{-1}(\kappa) P_{k} \beta_{0} \alpha \log _{2} e\left(\delta_{k}(q)-\delta_{k}\left(q^{l}\right)\right)}{2\left(\gamma H^{2}+\delta_{k}\left(q^{l}\right)\right)\left(\left(\gamma H^{2}+\delta_{k}\left(q^{l}\right) \Gamma\right)^{\alpha / 2}+\operatorname{Pr}^{-1}(\varepsilon) P_{k} \beta_{0}\right)}
\end{aligned}
$$

According to [14], it can be known that the first-order Taylor expansion of the convex function is a global low estimate. Assuming that the transmission power is set to 0 after the first-order Taylor expansion at a certain point, $R_{k}[m] \geq R_{\mathrm{kl}}^{\mathrm{inf}}[\mathrm{m}]$ can be obtained. According to the paper [1], the problem can be optimized as:

$$
\left(P 3^{\prime}\right): \quad \max _{Q, \varsigma} \varsigma^{\text {inf }} \quad \text { s.t. } \frac{1}{r_{k}} \sum_{m=1}^{M} a_{k}[m] R_{k, l}^{\text {inf }}[m] \geq \varsigma^{\text {inf }}, \quad \forall k,
$$

In this problem, $\varsigma^{\mathrm{inf}}$ is a slack variable that needs to be maximized, and $R_{\mathrm{kl}}^{\mathrm{inf}}$ is a concave quadratic function related to $q[\mathrm{~m}]$. Therefore, the $P 3$ problem is a convex function and can be solved with the CVX tool.

\section{3) The overall algorithm can be summarized as follows:}

Since the relaxation problem is not jointly convex for $X$ and $Q$, we use the block coordinate descent technique to solve $X$ and $Q$ alternately. When performing the $(I+1)$-th alternate solution, assign $Q_{1}$ at this time to the trajectory of the P2 problem to find $X_{l+1}$. Then bring $X_{l+1}$ into the $P 3$ problem and get $Q_{l+1}$ at this time through optimization. When it is judged that the loss increase in the $P 1$ problem is less than a certain value, the optimization is completed. For the solution of the $P 2$, because the weighted minimum throughput of the $P 3$ is maximized, making the constraint (7) relaxed. So more optimization space is left for the reduction of $\theta$ in $P 2$. The objective function of $P 2$ will not increase and the solution algorithm is convergent. For the solution of the $P 3$, its objective function value does not decrease in iterations, and the solution algorithm guar- 
antees convergence.

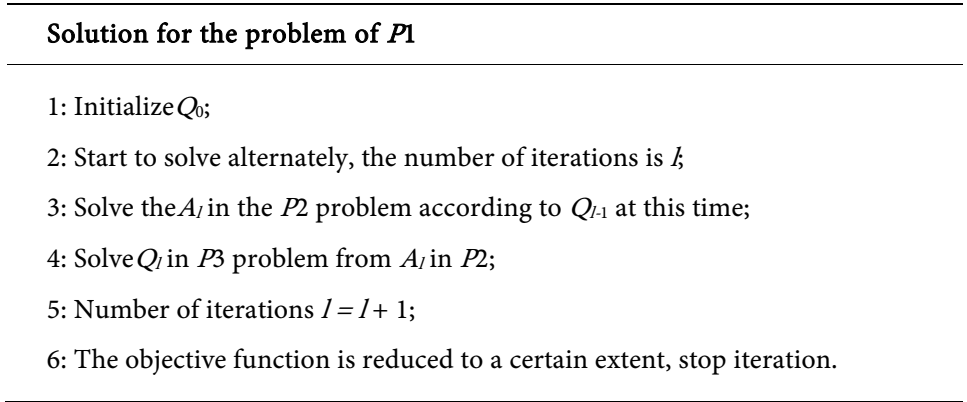

\section{Simulation and Results}

In this section, numerical results are given to verify the proposed design. The simulation runs on a 64-bit processor computer: Intel(R) Core(TM) i5-7200U CPU @ $2.50 \mathrm{GHz} 2.70 \mathrm{GHz}$. It is assumed that ten nodes are randomly generated within a range of $1600 \mathrm{~m} * 1600 \mathrm{~m} . q_{0}$ is $(-800,0)$ and $q_{F}$ is $(800,0)$. The UAV flies from a fixed height of $H=100 \mathrm{~m}$. It starts from the initial point and arrives at the end point to collect data. The entire data collection process assumes that the time is $T=50 \mathrm{~s}$. Furthermore, we set $V_{\max }=50 \mathrm{~m} / \mathrm{s}, \Delta t=0.5 \mathrm{~s}, B=1 \mathrm{MHz}, \beta_{0}$ $=-60 \mathrm{~dB}, \sigma^{2}=-120 \mathrm{dBm}, \Lambda=8 \mathrm{~dB}, \alpha=2, \varepsilon_{1}=0.001, \varepsilon_{2}=0.01, P_{k}=0.2 \mathrm{~W}, S_{k}=$ 10 Mbits and $\kappa=0.01$. When the result of each iteration does not increase more than 0.0001 , the optimization ends.

According to the Rice fading channel distribution, $K$ is used as the Rice factor. In this paper, we assume that the Rice factor $K=10$. According to [15], the cumulative distribution function can be expressed as:

$$
\operatorname{Pr}\left(\frac{\sigma^{2}\left(2^{R_{k}[m]}-1\right) \Lambda}{\beta_{k}[m] P_{k}}\right)=1-Q\left(\sqrt{2 K}, \sqrt{2(K+1) \frac{\sigma^{2}\left(2^{R_{k}[m]}-1\right) \Lambda}{\beta_{k}[m] P_{k}}}\right)
$$

where, the $Q$ function represents the Marcum $Q$ function defined by the first kind of zero-order modified Bessel function, which can be expressed in the following form:

$$
Q(a, b)=\int_{b}^{\infty} x \exp \left(-\frac{a^{2}+x^{2}}{2}\right) I_{0}(a x) d x
$$

In Figure 2, it shows the optimization results of drone flight trajectory when at most $1, \ldots, 5 \mathrm{SN}$ are connected at the same time. It can be observed that the trajectory when $n=1$ is obviously different from when $n$ is other values. The trajectory is almost the same when accessing more than two nodes at the same time.

In Figure 3, it shows the wake-up scheduling scheme where ten randomly generated SNs access at most one SN to upload data at the same time. According to the image, it can be seen that at most one node can upload data at the same time. It shows the scheduling scheme of ten randomly generated SNs simultaneously accessing at most two SNs in Figure 4. It can be seen that two SNs are connected at the same time. 


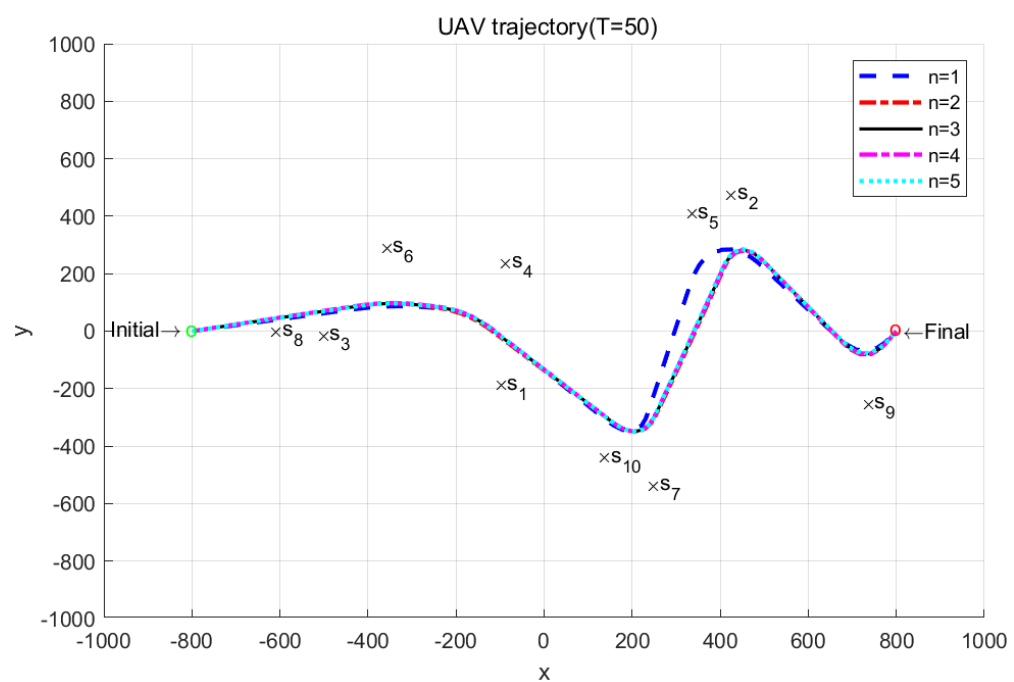

Figure 2. UAV flight path

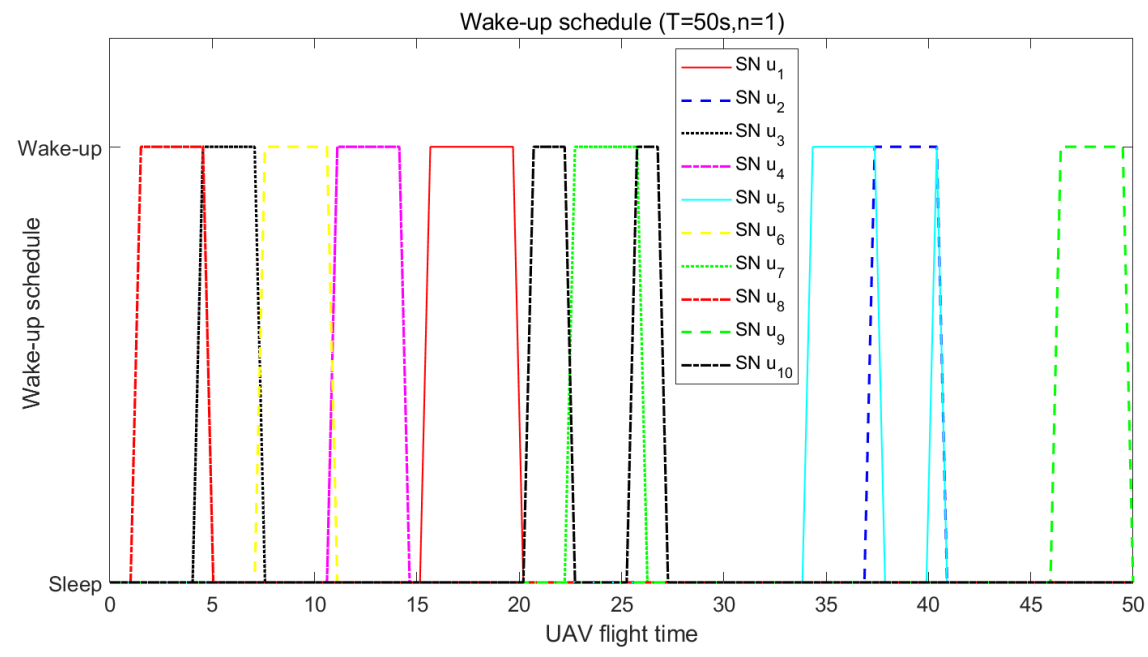

Figure 3. Wakeup scheme when $n=1$.

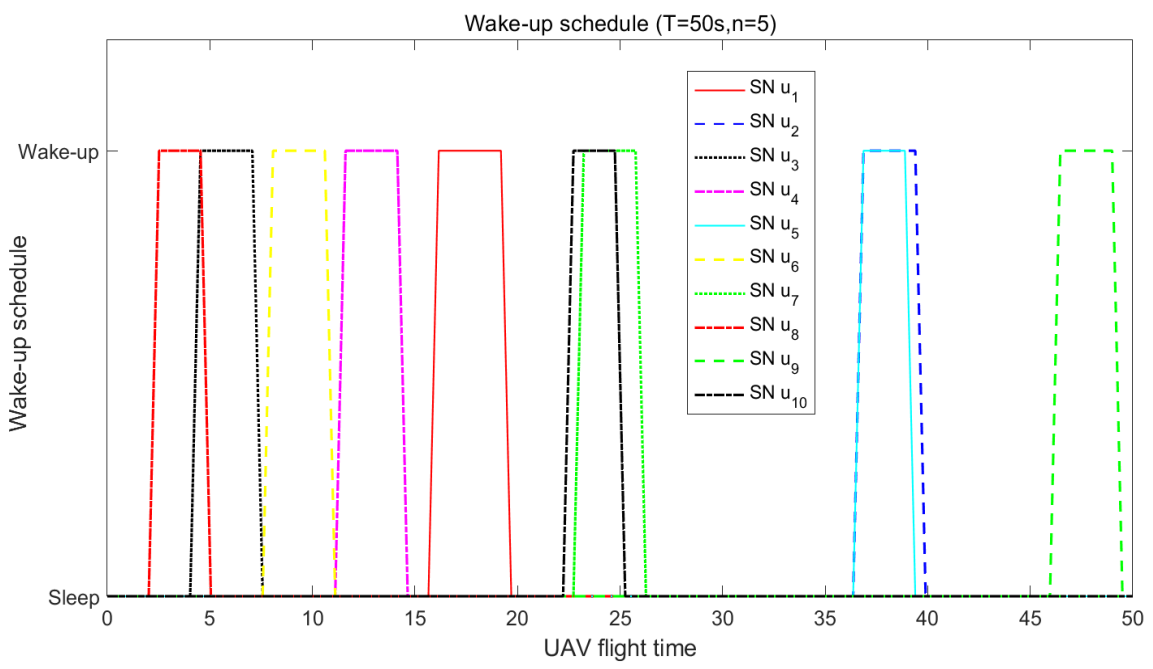

Figure 4. Wakeup scheme when $n=5$. 
Table 1. The optimal time for randomly generating ten nodes to access at most $n$.

\begin{tabular}{cccccc}
\hline$n$ & 1 & 2 & 3 & 4 & 5 \\
time $(\mathrm{s})$ & $38,420.34$ & $24,830.11$ & $22,590.62$ & $18,494.69$ & $19,928.12$ \\
\hline
\end{tabular}

Table 2. The loss of each node of ten randomly generated nodes.

\begin{tabular}{ccccccccccc}
\hline$\theta$ & $k=1$ & $k=2$ & $k=3$ & $k=4$ & $k=5$ & $k=6$ & $k=7$ & $k=8$ & $k=9$ & $k=10$ \\
\hline$n=1$ & 64.07 & 63.53 & 63.30 & 63.55 & 63.57 & 63.56 & 63.50 & 64.51 & 63.56 & 63.63 \\
$n=2$ & 64.96 & 64.48 & 65.06 & 64.51 & 64.58 & 64.56 & 64.49 & 65.20 & 64.57 & 64.84 \\
$n=3$ & 65.13 & 64.69 & 65.27 & 64.74 & 64.81 & 64.77 & 64.69 & 65.40 & 64.77 & 65.07 \\
$n=4$ & 65.17 & 64.72 & 65.29 & 64.78 & 64.84 & 64.80 & 64.72 & 65.43 & 64.80 & 65.09 \\
$n=5$ & 65.35 & 64.87 & 65.48 & 64.91 & 65.01 & 64.94 & 64.86 & 65.61 & 64.94 & 65.27 \\
\hline
\end{tabular}

Table 1 shows the optimization time when $n=1, \ldots, 5$ respectively. It can be found that the optimization time is the longest when at most one SN is connected. $n=4$ and $n=5$ corresponds to the optimal and suboptimal time respectively. When the value of $n$ is greater than 1, the optimization time will be cut back.

Table 2 shows the efficiency of each node after the optimization. It can be found that the efficiency is generally low when at most one node is connected.

\section{Conclusion}

This paper proposes an energy-saving scheme based on sparse access to optimize data collection between SNs and UAV. It is mainly by randomly generating ten ground nodes, jointly optimizing the trajectory of the UAV and the wake-up scheme of the SNs to minimize the data transmission loss. For the same number of SNs with the same distribution location, no more than $n$ nodes can be used for data transmission at the same time. When the value of $n$ is greater than 1 , the optimization time and transmission efficiency are usually better than the value of 1 . This means that the optimization algorithm proposed in this paper will improve the time efficiency and transmission efficiency.

\section{Acknowledgements}

This work was supported by the Research Fund for Young Teachers of Minzu University of China (2021QNPY109).

\section{Conflicts of Interest}

The authors declare no conflicts of interest regarding the publication of this paper.

\section{References}

[1] Zhan, C., Zeng, Y. and Zhang, R. (2018) Energy-Efficient Data Collection in UAV Enabled Wireless Sensor Network. IEEE Wireless Communications Letters, 7, No. 3, 
328-331. https://doi.org/10.1109/LWC.2017.2776922

[2] Zhang, G., Yan, H., Zeng, Y., Cui, M. and Liu, Y. (2018) Trajectory Optimization and Power Allocation for Multi-Hop UAV Relaying Communications. IEEE Access, 6, 48566-48576. https://doi.org/10.1109/ACCESS.2018.2868117

[3] Chen, Y., Feng, W. and Zheng, G. (2018) Optimum Placement of UAV as Relays. IEEE Communications Letters, 22, No. 2, 248-251. https://doi.org/10.1109/LCOMM.2017.2776215

[4] Al-Hourani, A., Kandeepan, S. and Lardner, S. (2014) Optimal LAP Altitude for Maximum Coverage. IEEE Wireless Communications Letters, 3, No. 6, 569-572. https://doi.org/10.1109/LWC.2014.2342736

[5] Mozaffari, M., Saad, W., Bennis, M. and Debbah, M. (2016) Unmanned Aerial Vehicle with Underlaid Device-to-Device Communications: Performance and Tradeoffs. IEEE Transactions on Wireless Communications, 15, No. 6, 3949-3963. https://doi.org/10.1109/TWC.2016.2531652

[6] Liu, B., Zhu, Q. and Zhu, H. (2020) Trajectory Optimization and Resource Allocation for UAV-Assisted Relaying Communications. Wireless Netw, 26, 739-749.

[7] Liu, B. and Zhu, H. (2019) Energy-Effective Data Gathering for UAV-Aided Wireless Sensor Networks. Sensors, 19, 2506.

[8] Zhan, C. and Huang, R. (2019) Energy Minimization for Data Collection in Wireless Sensor Networks with UAV. 2019 IEEE Global Communications Conference (GLOBECOM), Waikoloa, HI, USA, 2019, 1-6. https://doi.org/10.1109/GLOBECOM38437.2019.9013148

[9] Yang, D., Wu, Q., Zeng, Y. and Zhang, R. (2018) Energy Tradeoff in Groundto-UAV Communication via Trajectory Design. IEEE Transactions on Vehicular Technology, 67, No. 7, 6721-6726. https://doi.org/10.1109/TVT.2018.2816244

[10] Hua, M., Wang, Y., Zhang, Z., Li, C., Huang, Y. and Yang, L. (2018) Power-Efficient Communication in UAV-Aided Wireless Sensor Networks. IEEE Communications Letters, 22, No. 6, 1264-1267. https://doi.org/10.1109/LCOMM.2018.2822700

[11] Zhan, C. and Zeng, Y. (2020) Aerial-Ground Cost Tradeoff for Multi-UAV-Enabled Data Collection in Wireless Sensor Networks. IEEE Transactions on Communications, 68, No. 3, 1937-1950. https://doi.org/10.1109/TCOMM.2019.2962479

[12] Li, J., et al. (2020) Joint Optimization on Trajectory, Altitude, Velocity, and Link Scheduling for Minimum Mission Time in UAV-Aided Data Collection. IEEE Internet of Things Journal, 7, No. 2, 1464-1475. https://doi.org/10.1109/JIOT.2019.2955732

[13] Zeng, Y. and Zhang, R. (2017) Energy-Efficient UAV Communication with Trajectory Optimization. IEEE Trans. Wireless Commun., 16, No. 6, 3747-3760.

[14] Boyd, S. and Vandenberghe, L. (2004) Convex Optimization. Cambridge Univ. Press, Cambridge, UK.

[15] Ono, F., Ochiai, H. and Miura, R. (2016) A Wireless Relay Network Based on Unmanned Aircraft System with Rate Optimization. IEEE Trans. Wireless Commun., 15, No. 11, 7699-7708. 